\title{
Journal of Contradicting Results in Science
}

\author{
I.E. Cock ${ }^{a b *}$ \\ Editor-In-Chief, Pharmacognosy Communications
}

${ }^{a}$ Environmental Futures Centre, Nathan Campus, Griffith University, 170 Kessels Rd, Nathan, Brisbane, Queensland 4111, Australia. ${ }^{b}$ Biomolecular and Physical Sciences, Nathan Campus, Griffith University, 170 Kessels Rd, Nathan, Brisbane, Queensland 4111, Australia.

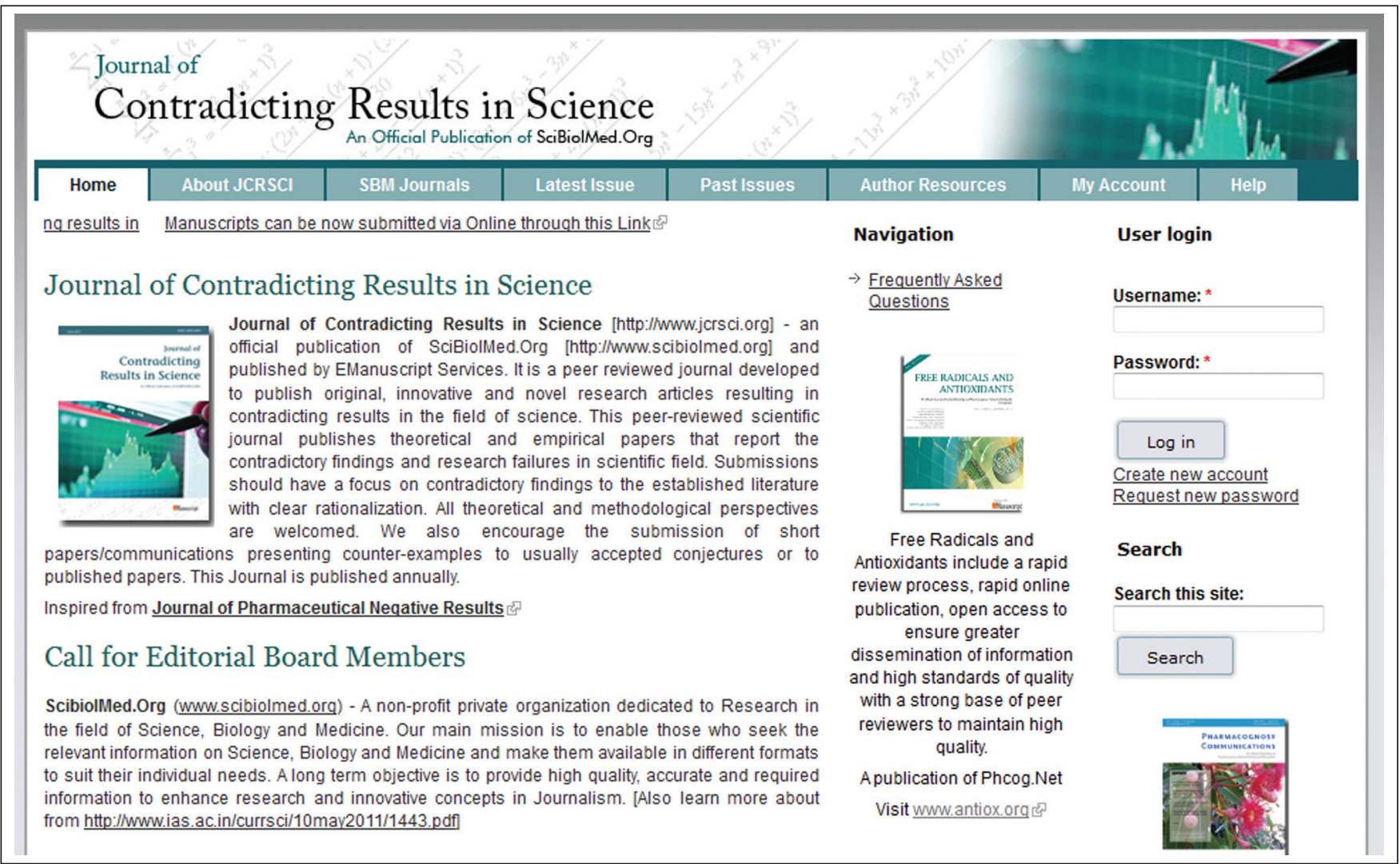

There is currently a bias to publish only results that are perceived as 'successful'; i.e. results that are positive (showing a significant finding) and/or that fit within the current model for a given research field. Results that are negative (supporting the null hypothesis) or are inconclusive are often not published, leading to a biased (perhaps false) representation in the published literature. Furthermore,

Correspondence:

Tel.: +61 737357637 ; fax: +61 737355282 .

E-mail: editor@phcogcommn.org; I.Cock@griffith.edu.au DOI: 10.5530/pc.2012.1.17 results that refute a current paradigm often meet with considerable resistance to publication. The Journal of Contradicting Results in Science (JCRSCI) (http:/ / www. jcrsci.org/) has been established by SciBiolMed.Org (http://www.scibiolmed.org) to address this imbalance. JCRSCI encourages the submission of original research articles resulting in controversial/negative results. By counterbalancing the selective reporting of 'positive' results, JCRSCI aims to foster discussion of controversial, unexpected and negative results and their context within current paradigms.

Much can be learned by examining why some ideas fail despite being well thought out and plausible. The publication of well documented contradictory results may highlight 
fundamental flaws in commonly used and accepted methods, reagents etc which may ultimately result in improvements in experimental design. The publication of negative or contradicting results may also avoid needlessly replicating fruitless experimental pathways. Alternatively, contradicting results may indicate new and interesting problems to be studied and may even ultimately result in paradigm shifts.

The scope of JCRSCI is broad. As well as covering pharmacognostic/pharmaceutical themes relevant to the readership of Pharmacognosy Communications, the journal also publishes contradictory results in the following general disciplines:

- Agricultural sciences

- Conservation and ecology

- Biological sciences including botany, zoology, microbiology, virology, cell biology, developmental biology, molecular biology

- Chronobiology and evolution

- Chemistry

- Physics and biophysics

- Geology

- Biotechnology

- Genetics and genomics expected to focus on results that contradict the established literature.

JCRSCI is a peer reviewed international journal which is expected to be published annually, with the first issue due in January 2012. The submission of short papers contradicting previous studies is also encouraged. Manuscripts published in JCRSCI will be peer reviewed for publication and are expected to be original and of high quality. The ideas tested in the publications are expected to be well explained and the reason they were tested well justified. Furthermore, to be meaningful, the contradicting results are expected to be explained, along with a discussion of the possible reason(s) why the study did not provide the predicted results and the possible implications of these findings.

It is apparent that journals for the publication of contradicting/negative results are needed. JCRSCI has potential to help fill this need and it is hoped that readers will support it by submitting their studies contradicting the established literature. Rigorous examination of balanced information (both positive and negative findings) may result in improvements in experimental design, and indicate new and important problems, as well as highlighting pitfalls and research dead ends. Therefore, far from reporting 'unimportant' findings, publication in journals such as JCRSCI may help stop researchers replicating redundant or fruitless experiments and in fact accelerate progress. It will be interesting to read the first issue of JCRSCI when it is released in 2012. 